\title{
Indeterminacy, Causality, and the Foundations of Monetary Policy Analysis
}

\author{
Bennett T. McCalluma
}

JEL-Classification: C61, C62, E37

Keywords: Causality, Indetermacy, Relational Expectations Equilibria

\section{Introduction}

The "New Monetary Policy Framework" of the Swiss National Bank, like that of most leading central banks, is frequently and appropriately studied by means of policy analysis conducted in the context of "New Keynesian" or "New Neoclassical Synthesis" models that presume rational expectations (RE) and utilize a policy rule for adjustment, in response to macroeconomic developments, of a specified short-term interest rate (e.g., the Swiss libor rate). This type of framework has become standard among monetary policy analysts, whether located in central banks or in academia, over the past 15 years. ${ }^{1}$

Throughout that period considerable attention has been given to issues relating to the possibility of "indeterminacy", meaning in this context a multiplicity of dynamically stable solutions. The standard approach to these issues has been to look for policy rules that yield, in the model adopted, "determinacy" in the sense of a unique stable RE solution - an approach developed in detail in the justly-influential treatise by WOODFORD (2003). There are several ways, however, in which this approach is arguably unsatisfactory. Cochrane (2007), for example, contends that a finding of determinacy - i.e., a single stable solution is not sufficient to imply a particular inflation outcome, for there exists in most cases a solution with explosive inflation that is not eliminated by the failure of

a Paper presented at the Swiss National Bank conference “The SNB's New Monetary Policy Framework Ten Years On”, held October 29-30, 2009, in Swiss Re Centre for Global Dialogue, Rüschlikon, Switzerland. The author is indebted to Katrin Assenmacher, Seonghoon Cho, Holger Sieg, and Cédric Tille for comments on earlier drafts.

1 For typical "three equation" formulations, see Woodford (2003, pp. 243-47), McCallum (2003, pp. 1159-61), and Cochrane (2007, pp. 23-27). 
any transversality condition. ${ }^{2}$ From a different perspective, McCallum (2003) argues that determinacy is not necessary for a unique solution to be implied because learnability [as developed in Evans and Honkapohja (2001)] is necessary for a solution to be plausible. In addition, Сно and McCALlum (2009) describe "another weakness" of determinacy as a selection criterion. ${ }^{3}$ Recently, in McCallum (2009a), I have argued for a different criterion for a RE solution to be plausible, namely, that it is fully consistent with the dynamic causality specification implied by the analyst's chosen structural model. In the present paper, I begin by summarizing this last argument. Then I recognize a weakness in the latter and put forth a proposed RE "solution refinement" that seems highly attractive. It is then shown that this refinement is consistent with the causality argument and adds support to a solution concept that was proposed over 25 years ago.

\section{Causality}

Here I begin by suggesting that there is an important sense in which indeterminate RE solutions reflect not a multiplicity of solutions for a single model, but instead a multiplicity of models each with a single solution. Specifically, it will be suggested that if attention is paid to the "direction of causality" of intertemporal relationships - expectational vs. inertial - then one of the RE solutions stands out uniquely as a candidate for equilibrium. In addition, structural models - e.g., ones based on optimizing analysis - typically require such attention in their specification. In developing this argument, I will add only one new ingredient to those standard in the RE literature; it constitutes an assumption of continuity of solution coefficients with respect to structural parameters (in the vicinity of zero).

The argument starts with the assertion that there can be little if anything more fundamental and critical, in dynamic economic modelling, than the specification and interpretation of the direction of causality in intertemporal relationships. For example, the univariate "model"

$$
y_{t}-\alpha y_{t+1}=0
$$

2 For an argument that this solution is, however, ruled out by the requirement of least-squares learnability, see McCallum (2009c).

3 This weakness is illustrated by an example in which there is a determinate solution that differs sharply in dynamic behavior from that implied by the (block recursive) model when considered on a sector-by-sector basis. 
can be interpreted as representing a system in which the variable $y_{t}$ is determined by agents' expectations in $t$ of $y_{t+1}$ or, alternatively, as representing a system in which $y_{t+1}$ is directly influenced by the previous period's realization of $y_{t}$ (or, equivalently, that $y_{t}$ is influenced by $\left.y_{t-1}\right)$. These two interpretations or specifications represent drastically different models of $y_{t}$ determination. ${ }^{4}$ The first features a crucial role for agents' current expectations of future values (with no influence from the past) whereas the latter assigns an impact to past values via, e.g., adjustment costs, and has no role for expectations (possibly because of an extreme discounting of the future). ${ }^{5}$ In terms of dynamic properties, to continue with the contrast, in the former case the system will be dynamically stable for any finite value of the parameter $a$, while the latter case features dynamic stability only if $|a|>1$. Also, simulations in stochastic versions with rational expectations are conducted quite differently in the two cases.

Accordingly, specifying the postulated direction of causation for all relations is an essential part of a model's specification, if that model is intended to represent the way in which data is generated by some economic system in which agents' expectations are potentially important. ${ }^{6}$ Of course it is the case that many - perhaps most - models include both expectational and inertial components. (These are the terms that will be used henceforth to refer to the two types of influences.) In such cases, the considerations just described remain fundamental; among other things, it is essential not to confuse parameter values relating to expectational components with those descriptive of adjustment-cost or other inertial aspects of the modeled mechanism. Any model that purports to be structural must surely be clear about all such distinctions. Indeed, all relevant causal specifications will be generated automatically in any model that is based on explicit analysis of agents' optimization problems plus market clearing (as, e.g., in socalled DSGE models).

How is causality specification accomplished, operationally, in the example under discussion, $y_{t}-\alpha y_{t+1}=0$ ? The answer is the same whether or not there

4 It should be clear that we are referring to "causality" in the model-specification sense of Simon (1953), not to Granger causality. For a useful discussion, see Zellner (1979), especially pp. 21-25.

5 In the first, the causality is not unidirectional; instead $y_{t}$ and expectations about $y_{t+1}$ are determined jointly. In the second, however, $y_{t-1}$ is not influenced by $y_{t}-$ it is predetermined.

6 If the first interpretation above is put forth for model (1), then there is no place for concern regarding dynamic stability. Observation of explosive tendencies in an empirical study of $y_{t}$ behavior would, accordingly, tend to discredit a hypothesis to the effect that the model with the first interpretation is appropriate for the data-generating mechanism at hand. 
is a stochastic component. The direction of causation is determined by specifying whether $y_{t}$ is or is not predetermined in relation (1) - i.e., unaffected by developments in the period to which the relationship pertains. ${ }^{7}$ Indeed, it will be shown in what follows that multiple solutions in linear rational expectations (RE) models invariably reflect multiple specifications regarding which variables are predetermined and which are not, and these specifications are in fact the operational counterpart of causality specifications. As the examples below will for simplicity exclude exogenous variables, the predetermined variables will in these cases also be the system's state variables.

The contention of the present argument is that recognition of the importance of causality specification, in the sense just described - i.e., of distinguishing expectational influences from inertial influences via adjustment costs, lags, etc. will, with the adoption of a simple and natural continuity property, eliminate issues relating to possible "indeterminacy" of the multiple-stable-solution type in linear rational expectations (RE) models. ${ }^{8}$ Specifically, there is in each model only one RE solution, which may be dynamically stable or unstable, that accurately reflects a given causality specification - that is, reflects a given specification of which variables in the system are predetermined. This solution might be regarded as representing a proposed equilibrium refinement; that position will be developed in Section 4. The continuity property that will be used in both arguments is that polynomials and eigenvalues relating to solution parameters are continuous functions of the model's structural parameters. Analysis involving such properties has a long and honorable history in economics, physics, and engineering. Some analysts may not be attracted by it, but many, I believe, will find it both attractive in principle and useful in practice.

\section{Basic Univariate Model}

Let us begin the discussion with a univariate linear model that features inclusion of both expectational and inertial influences, assuming of course that the analyst specifies which is which. ${ }^{9}$ That is, in the model

7 If $y_{t}$ is taken to be predetermined in (1) it might be natural to write the relation as (1/a) $y_{t}-y_{t+1}=0$ or, equivalently, $y_{t}=(1 / a) y_{t-1}$.

8 The importance and prevalence of such issues in monetary economics is stressed in MCCALLUM (2003).

9 It is possible, of course, to exclude one influence or the other by having either a or c equal to zero. 


$$
y_{t}=\gamma+a E_{t} y_{t+1}+c y_{t-1},
$$

inclusion of the $E_{t}$ operator before $y_{t+1}$ indicates that the analyst has specified that $\mathrm{a}$ is the parameter that governs the magnitude of expectational influences of $E_{t} y_{t+1}$ on $y_{t}$ while $c$ is the parameter governing inertial effects of $y_{t-1}$ on $y_{t}{ }^{10}$ It is crucial to recognize that in this framework agents are depicted as looking into the future while taking proper account of both expectational and inertial effects. ${ }^{11}$ We could add exogenous variables, stochastic or deterministic, to this equation but doing so would have no significant influence on the argument. The only state variable is $y_{t-1}$ (which is predetermined) so the fundamental (sunspotfree) forward-looking linear solution is of the form

$$
y_{t}=\phi_{0}+\phi_{1} y_{t-1}
$$

Accordingly, $E_{t} y_{t+1}=\phi_{0}+\phi_{1}\left(\phi_{0}+\phi_{1} y_{t-1}\right)$ and simple undetermined-coefficient reasoning indicates that $\phi_{0}$ and $\phi_{1}$ must satisfy

$$
\begin{gathered}
\phi_{0}=\gamma+a \phi_{0}+a \phi_{1} \phi_{0} \\
\phi_{1}=a \phi_{1}^{2}+c .
\end{gathered}
$$

For a given value of $\phi_{1}$, (4) determines $\phi_{0}$ uniquely but, obviously, (5) is satisfied by two values, which are

$$
\begin{aligned}
\phi_{1}^{(-)} & =\frac{1-\sqrt{1-4 a c}}{2 a} \\
\phi_{1}^{(+)} & =\frac{1+\sqrt{1-4 a c}}{2 a} .
\end{aligned}
$$

10 Then, a fully inertial specification with no significant role for expectations, i.e., $a=0$, has as its solution $y_{t}=\gamma+c y_{t-1}$, not $y_{t}=(1 / a)\left(y_{t-1}-c y_{t-2}\right)$.

11 It reflects a solution that BLANCHARD (1979, p. 115) describes as “... a weighted average of two special solutions, a backward solution ... and a forward solution...”. Our argument is that careful attention to the model's causality properties results in a unique determination of the weights assigned to these special solutions. The lag-operator approach of SARGENT (1979) is similar to, but more complete than, BLANCHARD's. 
We now ask, is there any connection between these two solutions and the correct identification of expectational and inertial components? Considering the special case in which $c=0$, so the inertial component is absent, we see that the answer is arguably "yes." For in that case, $\phi_{1}^{(-)}=0$ whereas $\phi_{1}^{(+)}=1 / a$. Thus the solution involving (6a) is appropriate whereas (6b) would suggest that causation is from $y_{t-1}$ to $y_{t}$ rather than from $E_{t} y_{t+1}$ to $y_{t}$.

That position is not accepted, however, by numerous analysts who take the position that expectations may depend on additional information variables, ones not included in the set of state variables implied by the model's specification. If, for example, $y_{t-1}$ is such a variable and is included even when $c=0$, then the conclusion that $\phi_{1}$ should equal zero when $c=0$ will not be accepted. More generally, this position argues for the eligibility as state variables "anything that agents decide to base their expectations on", including sunspot variables", ones unrelated to the model at hand.

Let us instead consider, therefore, the situation in which $a=0$, i.e., in which the importance of expectations in model (2) is nil. That is, we consider the contrasting special case in which $\mathrm{c}$ is non-zero but a equals zero. Then from (6b) we see that as $a \rightarrow 0$, we have $\phi_{1}^{(+)} \rightarrow \pm \infty$. By contrast, l'Hospital's rule shows that $\phi_{1}^{\left({ }^{(-)}\right.}$ approaches c. ${ }^{12}$ Thus we find that for this special case, as well as the one with $c=0, \phi_{1}^{(-)}$provides the a-priori correct value while $\phi_{1}^{(+)}$implies a value that is incorrect in the sense of departing from the causality specification that has been built into the model. Furthermore, the two expressions for $\phi_{1}$ are continuous functions of the basic parameters a and c of the model's structural relations. Thus for values of either $\mathrm{c}$ or a close to zero, the dynamic properties of the system, as determined by the value of $\phi_{1}$, will be close to those known to be relevant if $\phi_{1}^{(-)}$ is adopted but not if $\phi_{1}^{(+)}$were chosen for $\phi_{1}{ }^{13}$ Accordingly, if we adopt the principle that the model's solution implies response functions that are continuous in the basic parameter values, we are justified in concluding that $\phi_{1}^{(-)}$provides the answer in general, i.e., it identifies the solution that correctly represents the causal structure implied by model (3), in which both expectational and inertial components are potentially present.

12 Both numerator and denominator of (6a) approach zero as $a \rightarrow 0$, but $d([1-\sqrt{1-4 a c}] / d a) \rightarrow 2 c$ while $(d[2 a] / d a)=2$, so the expression in (6a) has a limiting value of $\mathrm{c}$.

13 This can be verified by numerical examples. Note for reference below that selection of the solution $\phi_{1}^{(-)}$simultaneously implies that $\phi_{1} \rightarrow 0$ as $c \rightarrow 0$ and that $\phi_{1} \rightarrow c$ as $a \rightarrow 0$, whereas the other solution has $\phi_{1}^{(+)} \rightarrow 1 / a$ as $c \rightarrow 0$ and $\phi_{1}^{(+)} \rightarrow \infty$ as $a \rightarrow 0$. 
In light of that contention, it is perhaps natural to ask, "to what causal dynamic specification does the solution $\phi_{1}^{(+)}$pertain?" The answer is reasonably straightforward. Suppose the analyst ignores the expectation operator $E_{t}$ in (2) and interprets the equation as a purely inertial model, writing it (with $\gamma=0$ ) as

$$
y_{t}=(1 / a) y_{t-1}-(c / a) y_{t-2} \text {. }
$$

Then for the special case with $c=0$ he finds $y_{t}=(1 / a) y_{t-1}$ as his solution, which is what he would find if he were using $\phi_{1}^{(+)}$. Generalizing to the case with $c \neq 0$, he might seek a solution to (2') that features only one state variable, i.e.,

$$
y_{t}=\psi y_{t-1} \text {. }
$$

The latter would imply also that $y_{t}=\psi^{2} y_{t-2}$, so substitution into (2') would imply that $\psi$ must satisfy $\psi^{2}=(1 / a) \psi-(c / a)$, which with $a \neq 0$ has the same form as (5). Accordingly, we see that (2') is a second interpretation that gives rise to the quadratic equation (5). In fact, ( $\left.2^{\prime}\right)$ is the relation that is associated with the root $\phi_{1}^{(+)}$, not only in the special case $c=0$, but also for cases with c close to zero, with a close to 0 , and indeed in general. Clearly, the causal structure is entirely different from that implied by the root $\phi_{1}^{(-)}$. The root $\phi_{1}^{(+)}$pertains to an interpretation of model (2) as if it were model (2'), which has a different dynamic specification.

In sum, the solution based on expression (6b) for $\phi_{1}$ is the solution to a model in which agents at time $t$ make decisions about $y_{t}$ on the basis of past values of $y_{t-1}$ and $y_{t-2}$, plus the constraint implied by (3'), with expectations of $y_{t+1}$ playing no role. ${ }^{14}$ This is quite different from the model specified in (2), which depicts agents as choosing $y_{t}$ values partly on the basis of $y_{t-1}$ (inertial influences) and also (potentially) on expectations regarding $y_{t+1}$. From a structural point of view, these are two drastically different models. But once the analyst has decided which of the two models he is proposing, there is no ambiguity about its solution. ${ }^{15}$

Before moving on, we note that in the simple univariate model at hand the values $\phi_{1}^{(-)}$and $\phi_{1}^{(+)}$equal the eigenvalues of the dynamic system written in first-

14 This is not a very sensible model, but it is what is implied by the solution using (6b). That is not at all a weakness for our argument, which by contrast posits (6a) as the appropriate expression.

15 This solution might still fail to provide an equilibrium because of the failure of some transversality condition or some informational feasibility condition such as least-squares learnability (as developed by extensively by Evans and Honkapohja (2001)). 
order form as well as possible values of the solution coefficient $\phi_{1}$ in (6). The analogous equivalence does not prevail in multivariate models, but in the latter the system's eigenvalues continue to govern and describe the model's dynamic stability properties. And it is well known that in a multivariate system eigenvalues are continuous functions of the basic parameters of the model's structural equations. ${ }^{16}$ Accordingly, it will be possible to relate different causal specifications of such a model to different groupings of system eigenvalues - groupings that imply different solutions for the multivariate counterpart of $\phi_{1}$.

Indeed, it is possible to generalize the conclusion obtained above - namely, that the appropriate RE solution can be identified as the one that results in a value of zero for the solution parameter $\phi_{1}$ when the structural parameter c equals zero by means of the multivariate counterpart of equation (2). It might be noted parenthetically that, in the univariate example above, this rule happens to coincide with adoption of the $\phi_{1}$ value that is the smaller in absolute value of the two that satisfy the quadratic (5). Such a coincidence does not always obtain, however, in systems with more endogenous variables; and in such cases the appropriate solution must be based on the procedure just described. The mechanics of this procedure is worked out in a companion paper, McCallum (2009a).

To complete the current discussion, however, let us consider "sunspot" solutions for model (2). These can be obtained by looking for solutions not of form (3) but more generally of form

$$
y_{t}=\phi_{0}+\phi_{1} y_{t-1}+\phi_{2} y_{t-2}+\phi_{3} \xi_{t}
$$

where $\xi_{t}$ is any stationary stochastic process that has the property $E_{t-1} \xi_{t}=0 .{ }^{17}$ To avoid unnecessary symbols, let us take $\phi_{0}=0$. Then we have

$$
E_{t} y_{t+1}=\phi_{1}\left(\phi_{1} y_{t-1}+\phi_{2} y_{t-2}+\phi_{3} \xi_{t}\right)+\phi_{2} y_{t-1}+0 \text {. }
$$

Substituting these two expressions into model (2) then gives

$$
\phi_{1} y_{t-1}+\phi_{2} y_{t-2}+\phi_{3} \xi_{t}=a\left[\phi_{1}\left(\phi_{1} y_{t-1}+\phi_{2} y_{t-2}+\phi_{3} \xi_{t}\right)+\phi_{2} y_{t-1}\right]+c y_{t-1} \text {. }
$$

16 See, e.g., Horn And Johnson (1985, pp. 539-540).

17 The multivariate version of (8) corresponds to the complete set of solutions considered in Sims (2002) and LubiK and SchorfHeide (2003). The latter authors apparently see it as desirable that Sims' method "... does not require the researcher to separate the list of endogenous variables ... into 'jump' and 'predetermined' variables" (2003, p. 276). The position of the present paper is basically just the opposite, assuming the desirability of having a structural model (which for Sims would perhaps not be the case). 
Accordingly, we have the undetermined-coefficient requirements

$$
\begin{gathered}
\phi_{1}=a \phi_{1}^{2}+a \phi_{2}+c \\
\phi_{2}=a \phi_{1} \phi_{2} \\
\phi_{3}=a \phi_{1} \phi_{3} .
\end{gathered}
$$

Now, the last two of these require that either $\phi_{1}=1 / \mathrm{a}$ or that $\phi_{2}=\phi_{3}=0$. But in the latter case we have the same solutions as in (6a) and (6b). In the former case, $\phi_{3}$ can be any number but then (11a) reduces to

$$
\frac{1}{a}=\frac{1}{a}+a \phi_{2}+c
$$

that is, to $\phi_{2}=-c / a$, which is not contradicted by (11b). So there is a sunspot solution

$$
y_{t}=\frac{1}{a} y_{t-1}-\frac{c}{a} y_{t-2}+\phi_{3} \xi_{t}
$$

for any value of $\phi_{3}$. For a suitable range of values of a and c, each of these solutions will be dynamically stable. But whether it is stable or unstable, a solution of form (8) is essentially a stochastic extension of (2') and thus reflects the same direction of causality as (3) with $\phi_{1}^{(+)}$from (6b) above, which we have suggested is inconsistent with the dynamic specification of the model (2). Thus sunspot expressions of the form implied by (8) with $E_{t-1} \xi_{t}=0$ are not candidates for equilibria for the model (2), given that a and $\mathrm{c}$ are its parameters pertaining to expectational and inertial influences, respectively. Equivalently, no sunspot expressions of the indicated form yield candidate equilibria for model (2) with the specification that $y_{t-1}$, but not $y_{t}$, is predetermined. Under that specification, a $\phi_{1} \neq 1$, $\phi_{2}=\phi_{3}=0$, and the relevant candidate solution is (3) with (6a). 


\section{Proposal as a Solution Refinement}

In this section the objective is to modify the foregoing argument by proposing a criterion or "solution refinement" based on continuity of solution coefficients with respect to structural parameters. The discussion will (as before) be limited to linear models.

Consider again the univariate model, assumed to be structural:

$$
y_{t}=a E_{t} y_{t+1}+c y_{t-1}, a \neq 1 .
$$

Here we have for simplicity omitted the constant term and exogenous shocks, which are inessential to the argument. The fundamental solutions are of the form

$$
y_{t}=\phi y_{t-1}
$$

so $E_{t} y_{t+1}=\phi^{2} y_{t-1}$. Then substitution of the latter and (15) into (14) followed by undetermined-coefficient (UC) reasoning indicates that $\phi$ must satisfy

$$
a \phi^{2}-\phi+c=0 .
$$

Thus the fundamental solutions are given by (14) with the following two values for $\phi$ :

$$
\begin{aligned}
\phi^{(-)} & =\frac{1-\sqrt{1-4 a c}}{2 a} \\
\phi^{(+)} & =\frac{1+\sqrt{1-4 a c}}{2 a} .
\end{aligned}
$$

The proposed refinement is that $\phi$ must be continuous in the parameters a and $\mathrm{c}$. In particular, $\phi$ must be continuous in 'a' over open intervals of values that include $(\mathrm{a}, \mathrm{c})$ and $(0,0)$. Then we focus on cases in which $\mathrm{a} \rightarrow 0$. The rationale is that in this extreme case expectational effects are absent so the solution is unambiguously $y_{t}=c y_{t-1}$. In addition, small values of 'a' reflect cases in which expectational effects are small, so they should imply solutions with $\phi$ close to c. Furthermore, continuity of solution parameters is necessary for impulse-response functions to be well behaved when exogenous variables are included in the model. 
Clearly, this requirement implies that the solution is given by (15) with the limiting value, as $a \rightarrow 0$, of $\phi^{(-)}=c$, as in (6a), and not by $\phi^{(+)}= \pm \infty$, i.e., an infinite discontinuity. ${ }^{18}$ It is useful to note also that (with $a \neq 0$ ) as the parameter $c \rightarrow 0$ we have $\phi^{(-)} \rightarrow 0$, whereas $\phi^{(+)} \rightarrow 1 / a$. Thus the refinement leads to the same solution as the minimum state variable (MSV) solution suggested by McCallum (1983).

From the foregoing we see that the proposed refinement leads to a single solution when we are limiting consideration to fundamental solutions. But suppose we admit solutions of the general "sunspot" form

$$
y_{t}=\phi_{1} y_{t-1}+\phi_{2} y_{t-2}+\phi_{3} \xi_{t}
$$

where $\xi_{t}$ is a stationary stochastic process with the property $E_{t} \xi_{t+1}=0 .{ }^{19}$ Then we have

$$
E_{t} y_{t+1}=\phi_{1}\left(\phi_{1} y_{t-1}+\phi_{2} y_{t-2}+\phi_{3} \xi_{t}\right)+\phi_{2} y_{t-1}+0
$$

and substitution into (14) leads to the following UC conditions:

$$
\begin{gathered}
\phi_{1}=a \phi_{1}^{2}+a \phi_{2}+c \\
\phi_{2}=a \phi_{1} \phi_{2} \\
\phi_{3}=a \phi_{1} \phi_{3} .
\end{gathered}
$$

Now, the last two of these require that either $a \phi_{1}=1$ or that $\phi_{2}=\phi_{3}=0$. In the latter case we have the same fundamental solutions as before, but in the former case $\phi_{3}$ can be any number and then (20a) reduces to

$$
\frac{1}{a}=\frac{1}{a}+a \phi_{2}+c
$$

that is, to $\phi_{2}=-c / a$, which is not contradicted by (20b). So there is a sunspot solution

18 The first of the two limits is obtained by means of l'Hôpital's rule.

19 Again it should be mentioned that any RE solution to model (14) can be expressed in this form. See, e.g., Lubik and SchorfHeide (2003). 


$$
y_{t}=\frac{1}{a} y_{t-1}-\frac{c}{a} y_{t-2}+\phi_{3} \xi_{t} .
$$

for any finite value of $\phi_{3}$. Clearly, however, the solution coefficients are not continuous at $a=0 .{ }^{20}$ So the proposed refinement rules out all solutions except (15) with $\phi$ given by $\phi^{(-)}$. Obviously, to be useful this result must extend to richer models. Even in the present context, however, it is interesting that there is only a single solution that satisfies the continuity principle. More significantly, perhaps, it is the same solution as the one that utilizes the "direction of causality" criterion as developed above in Sections 2 and 3, as well as the "minimum state variable" solution. In any event, it is the case that the above argument carries over to a multivariate version of model (14); this is demonstrated in MCCALLum (2009b).

\section{Concluding Remarks}

For the argument developed above to be of broad significance, it must - as just suggested - be extended to a more general multivariate setting. But the needed extension is available. Specifically, in McCallum (2009a, 2009c) it is shown that a generalization of the results applies to any model that can be written in the following form:

$$
\begin{gathered}
y_{t}=\mathrm{AE}_{t} y_{t+1}+\mathrm{C} y_{t-1}+\mathrm{D} u_{t} \\
u_{t}=\mathbf{R} u_{t-1}+\varepsilon_{t} .
\end{gathered}
$$

Here $\mathbf{A}$ and $\mathbf{C}$ are $m \times m, \mathbf{D}$ is $m \times n, \varepsilon_{\mathrm{t}}$ is $n \times 1$ white noise, and $\mathbf{R}$ is $n \times n$ with all eigen-values less than 1 in modulus. This specification is very broad; any model satisfying the forms used by KING and WATson (1998) or KLEIN (2000) can be written in this manner, thereby admitting any finite number of lags, expectational leads, and lags of leads.

In sum, then, the present paper proposes a solution refinement that yields a single rational expectations solution for a very broad class of linear models including those generally used in monetary policy analysis. This result is of major importance for obtaining the practical implications of standard monetary policy

20 The coefficient on $y_{t-1}$ in (22) implies that a tiny change in the expectational parameter a say, from 0.01 to -0.01 - could have an implausibly large effect on the dynamic behavior of $y_{t}$. 
analysis in a variety of practical policy concerns. Examples of the latter include issues relating to the Taylor principle, inflation forecast targeting, the deflationtrap hypothesis, and the fiscal theory of the price level.

\section{References}

Blanchard, Olivier J. (1979), "Backward and Forward Solutions for Economies with Rational Expectations", American Economic Review, 69, pp. 114-118.

Blanchard, Olivier J., and Charles M. Kahn (1980), "The Solution of Linear Difference Models Under Rational Expectations", Econometrica, 48, pp. 1305-1311.

Calvo, Guillermo (1978), "On the Indeterminacy of Interest Rates and Wages with Perfect Foresight”, Journal of Economic Theory, 19, pp.321-337.

Cho, Seonghoon, and Bennett T. McCallum (2009), "Another Weakness of 'Determinacy' as a Selection Criterion for Rational Expectations Models", Economics Letters, 104, pp. 17-19.

Cochrane, John H. (2007), "Inflation Determination with Taylor Rules: A Critical Review", NBER Working Paper 13409.

Evans, George W., and Seppo Honkapohja (2001), Learning and Expectations in Macroeconomics, Princeton.

Golub, Gene H., and Charles F. Van Loan (1996), Matrix Computations, 3rd ed. Baltimore.

Horn, Roger A., and Charles R. Johnson (1985), Matrix Analysis, New York.

Horn, Roger A., and Charles R. Johnson (1991), Topics in Matrix Analysis, Cambridge University Press.

King, Robert G., and Mark W. Watson (1998), "The Solution of Singular Linear Difference Systems Under Rational Expectations”, International Economic Review, 39, pp. 1015-1026.

Klein, Paul (2000), "Using the Generalized Schur Form to Solve a Multivariate Linear Rational Expectations Model", Journal of Economic Dynamics and Control, 24, pp. 1405-1423.

Lubik, Thomas A., and Frank Schorfheide (2003), "Computing Sunspot Equilibria in Linear Rational Expectations Models", Journal of Economic Dynamics and Control, 28, pp. 273-285.

McCallum, Bennett T. (1983), "On Nonuniqueness in Linear Rational Expectations Models: An Attempt at Perspective", Journal of Monetary Economics, 11, pp. 139-168. 
McCallum, Bennett T. (2003), "Multiple-Solution Indeterminacies in Monetary Policy Analysis", Journal of Monetary Economics, 50, pp. 1153-1175.

McCallum, Bennett T. (2009a), "Causality, Structure, and the Uniqueness of Rational Expectations Equilibria”, NBER Working Paper 15234.

McCallum, Bennett T. (2009b), "A Continuity Refinement for Rational Expectations Solutions”, Working Paper, Carnegie Mellon University.

McCallum, Bennett T. (2009c), "Inflation Determination with Taylor Rules: Is New-Keynesian Analysis Critically Flawed?", Journal of Monetary Economics, 56, pp. 1101-1108 and 1114-1115.

Sargent, Thomas J. (1979) Macroeconomic Theory, Academic Press (2nd edition 1987).

Simon, Herbert A. (1953) "Causal Ordering and Identifiability", in W.C. Hood and T.C. Koopmans (eds), Studies in Econometric Method, Cowles Commission Monograph No. 14, New York.

Sims, Christopher A. (2002), "Solving Linear Rational Expectations Models", Computational Economics, 20, pp. 1-20.

Uhlig, Harald (1999), "A Toolkit for Analyzing Nonlinear Dynamic Stochastic Models Easily", in Ramon Marimon and Andrew Scott (eds), Computational Methods for the Study of Dynamic Economies, Oxford.

Zellner, Arnold (1979) "Causality and Econometrics", Carnegie-Rochester Conference Series on Public Policy 10, pp.9-54.

\section{SUMMARY}

To be useful as a guide to behavior, a model that includes a relationship between $x_{t}$ and $z_{t+1}$ must specify whether $x_{t}$ is influenced by the expectation at $t$ of $z_{t+1}$ or, that $z_{t+1}$ is inertially influenced by $x_{t}$. We show that, for a broad class of linear RE models, distinct causal specifications will be uniquely associated with distinct solutions. Alternatively, a solution refinement requiring continuity of solution coefficients with respect to basic parameters implies this same solution. For a given structure there is only one RE solution that is fully consistent with the model's specification. 